\title{
Radial versus right internal thoracic artery as a second arterial conduit for coronary surgery: Early and midterm outcomes
}

Bruce W. Lytle, MD

See related article on page 39 .

From the Department of Thoracic and Cardiovascular Surgery, The Cleveland Clinic Foundation, Cleveland, Ohio.

Received for publication Dec 20, 2002; accepted for publication Feb 11, 2003.

Address for reprints: Bruce W. Lytle, MD, Department of Thoracic and Cardiovascular Surgery, The Cleveland Clinic Foundation, 9500 Euclid Ave, \#F-25, Cleveland, OH 44195 (E-mail: lytleb@ccf.org).

J Thorac Cardiovasc Surg 2003;126:5-6

Copyright ( $\odot 2003$ by The American Association for Thoracic Surgery

$0022-5223 / 2003 \$ 30.00+0$

doi:10.1016/S0022-5223(03)00596-8

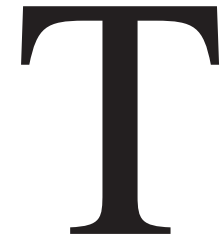

he left internal thoracic artery (LITA) to left anterior descending coronary artery (LAD) graft is now a fundamental part of operations for coronary revascularization. There is a mountain of observational evidence that this strategy produces short- and long-term outcomes that are superior to a vein graft-only strategy. The LITA-LAD graft has higher early and later patency rates than vein grafts and decreases the risk of early and late death. A lesser, but increasingly substantial, body of evidence from multiple institutions appears to show that bilateral ITA grafting improves outcomes relative to a single ITA graft strategy. Reactivation of the use of the radial artery (RA) as a bypass graft has provided the option of substituting the RA for the right internal thoracic artery (RITA) as a second arterial bypass graft.

There are many potential advantages of using the RA instead of the RITA graft. The RA is larger and easier to work with than the RITA, its preparation is straightforward, can be accomplished during the LITA dissection, and using the RA instead of the RITA avoids any increase in sternal wound complications associated with bilateral ITA grafting. However, it is not known whether substituting the RA for the RITA as a second arterial graft provides similar revascularization outcomes to a bilateral ITA strategy. Is the RA as good a coronary bypass graft as the RITA? The article by Caputo and associates ${ }^{1}$ in this issue of the Journal attempts to shed light on this question.

In their retrospective observational study, short-term (mean follow-up 18 months) clinical outcomes are compared for patients receiving LITA-RA grafts with those for patients receiving LITA-RITA grafts. The conclusion of the authors was that, in their hands, the RA graft was not only as good a graft as the RITA but that it produced better short-term outcomes. Patients with RA grafts had fewer perioperative events (in particular, fewer blood transfusions and episodes of atrial fibrillation) and a lower risk of early out-of-hospital events, primarily because of a lower rate of recurrent angina. This is interesting information and reflects the experience of that center. However, this study does not yet provide the kind of information that would allow us to reach the general conclusion that the RA is a superior bypass graft to the RITA.

According to the authors, only $15 \%$ of their patients undergoing revascularization during the time period of the study received two arterial bypass grafts of any kind, indicating that this center was not heavily committed to the use of arterial grafts at that time. Some of the adverse outcomes of the patients who received RITA grafts, in particular myocardial infarction and return of angina, may have been technically related rather than being related to the intrinsic ability of the graft to stay patent and relieve ischemia. These are short-term ischemic outcomes. In our study that compared bilateral versus single ITA grafting, in essence testing the RITA versus vein grafts, differences in clinical outcomes did not become apparent until about 6 years after the operation, when vein graft failure due to intimal fibroplasia and graft atherosclerosis begins to appear. The outcomes reported by Caputo and associates were essentially perioperative. Most surgeons would agree that the use of the RITA is technically more demanding than is the use of the RA, but as experience with arterial grafting increases, the technical complications of RITA grafting may di- 
minish. Also, the authors point out that the RITA was commonly used as an in situ graft to the right coronary artery, a strategy subject to a higher risk of graft failure than other graft-coronary artery combinations. Although they note that this is a problem with their analysis and state that statistical adjustments were made, the number of patients and adverse events were relatively small and it is unclear what those adjustments could be.

Two questions still remain. Does the incremental technical difficulty of RITA grafting produce a fixed rate of graft attrition that will be difficult to overcome? And, does the RITA possess a better intrinsic tendency to stay patent over the long term compared with the RA? Although we have an enormous amount of information available regarding the patency of the LITA-LAD graft, less data exists involving the use of either the RITA or LITA to other vessels, and those data that we do have indicate inferior patency rates to the LITA-LAD gold standard. We do know that RITA grafts are capable of remaining patent for 20 years without becoming atherosclerotic. Studies of RA grafts demonstrate perfect patency rates of $80 \%$ to $87 \%$ at 5 years, and anecdotally we and others have noted radial artery graft patency at 8 to 10 years after operation. Both RITA and RA grafts have diminished patency rates when grafted to coronary vessels that have mild or moderate obstructions.

My opinion regarding the answers to the two questions is that the technical costs of RITA grafting do diminish with experience and that given a "perfect" situation (a graft to a tightly stenotic branch of the left coronary system) the RITA will have a better long-term patency rate than an RA graft. That opinion is based on a number of somewhat anecdotal observations. (1) There is a high rate of success with the RITA when used in this "perfect" situation. (2) We know that RITA grafts can continue to be patent for at least 20 years after operation. (3) We know that when the LITA is used as a graft to the LAD, it is not only possible but probable that it will function well more than 20 years after the operation; it is hard to understand what fundamental difference there would be between the LITA and the RITA in this regard. (4) My colleagues and I have noted anecdotal occlusions of RA grafts placed in "perfect" situations that are hard to understand. There are certainly many RA grafts that have functioned well in those "perfect" situations, but even anecdotal occlusions are somewhat disturbing. In my judgment, the RA graft is less predictable than the RITA graft in regard to patency. I am painfully aware that these opinions are not conclusions based entirely on statistically significant randomized or even prospective data, but they are my opinions at this time.

The RA is clearly a useful graft and many patients will benefit from both RA and RITA grafts. The advantages and disadvantages of both will depend on patient-related, conduit-related, coronary artery-related, and surgeon-related variables. Currently I personally use the RITA in preference to the RA for most patients. The study of Caputo and associates provides important information and is a good point of reference from which to start, but this is an important question necessitating much more data.

\section{Reference}

1. Caputo M, Reeves B, Marchetto G, Mahesh B, Lim K, Angelini GD. Radial versus right internal thoracic artery as a second arterial conduit for coronary surgery: early and midterm outcomes. J Thorac Cardiovasc Surg. 2003;126:39-47. 